October 23, 2000

KEK-TH-717

DAMTP-2000-115

\title{
Open Superstring Theory and Superalgebra of the Brane Antibrane System
}

\author{
Machiko Hatsuda and Makoto Sakaguchi ${ }^{\dagger}$ \\ Theory Division, High Energy Accelerator Research Organization (KEK), \\ Tsukuba, Ibaraki, 305-0801, Japan \\ $\dagger$ Department of Applied Mathematics and Theoretical Physics \\ Wilberforce Road, Cambridge, CB3 OWA, U.K. \\ E-mail: mhatsuda@post.kek.jp, M.Sakaguchi@damtp.cam.ac.uk
}

\begin{abstract}
Open superstring theory is formulated in terms of a nondegenerate supertranslation algebra. A supercharge for a tachyonic superstring can be also defined classically by taking into account the leakage of the supercurrent which is compensated by fermionic and bosonic auxiliary fields. The anticommutator of two supercharges of the tachyonic superstring does not contain the zero eigenvalue and so this string is not a BPS state. Brane-antibrane annihilation scenarios are described by these superalgebras defined on the sum of world-volumes of a D-brane, an anti-D-brane and a tachyonic superstring.
\end{abstract}

PACS: 11.17.+y; 11.30.Pb

Keywords: Superalgebra; SUSY central extension; BPS states; D-brane 


\section{Introduction}

The classification of states according to their transformation by elements of the superalgebra is a powerful tool for exploring non-perturbative aspects of supersymmetric theories [1]. The anticommutator of two supercharges is a real symmetric matrix

$$
\left\{Q_{\alpha}, Q_{\beta}\right\}=M_{\alpha \beta}
$$

States are classified by the matrix $M_{\alpha \beta}$ as follows:

1. If all eigenvalues of $M_{\alpha \beta}$ are zero, the state preserves all supersymmetries. This is the case for the ground states of fundamental superstrings, i.e. the vacuum state which has no strings.

2. If at least one of eigenvalues of $M_{\alpha \beta}$ is zero, the state preserves some supersymmetries. This is a BPS state.

3. If all eigenvalues of $M_{\alpha \beta}$ are non-zero, then all supersymmetries are broken. This is a non-BPS state.

As well as BPS systems [2, 3], recent developments of non-BPS systems have lead to the understanding of a variety of non-perturbative phenomena in string theories $14,6,6,6,8$, 9, 10, 11, 12.

Although space-time supersymmetry (SUSY) is essential, most studies of this field are developed by using the Neveu-Schwarz-Ramond (NSR) formalism rather than the Green-Schwarz (GS) formalism where space-time SUSY is manifest. In order to represent space-time SUSY in the NSR formalism a GSO projection is required, and it plays an essential role in non-BPS physics. The wrong GSO projection results in tachyonic modes and instability of the system [8]. Recently it was pointed out that tachyonic modes are obtained in the GS formalism by the "wrong" boundary condition by looking at the partition function [13]. In this paper we try to explain appearance of tachyonic modes in a GS like action. Our guiding principle is the global SUSY of the action, where SUSY is broken by the wrong boundary condition. The problem is how to represent the non-BPS superalgebra for a tachyonic superstring and how to recover SUSY.

In order to consider a tachyonic superstring it is important to reexamine open string theory. In the Kalb and Ramond discussion of the open string interaction [14] the open string current is not conserved, and leaks from the end points. The open string gauge interaction is not gauge invariant, but gauge invariance is recovered by introducing a vector gauge field coupling with the endpoint currents. For the type II superstring theories the corresponding gauge invariant interaction is

$$
I_{W Z}=\frac{1}{2} \int_{\Sigma} B^{N S}-\int_{\partial \Sigma} A=\frac{1}{2} \int_{\Sigma} \mathcal{B} \quad, \quad \mathcal{B}=B^{N S}-d A
$$

where $\Sigma$ is a string world-sheet, $\partial \Sigma$ is its boundary, $B^{N S}$ is the Neveu-Schwarz two-form, and $A$ is the Dirac-Born-Infeld (DBI) vector gauge field on Dp-branes [2]. This WessZumino action is not only gauge invariant but also SUSY invariant. The manifestly SUSY 
invariant Wess-Zumino action can be obtained as an element of the trivial class of the Chevalley-Eilenberg cohomology of the nondegenerate supertranslation group [15, 16]. The nondegenerate supertranslation group has been examined recently in many kinds of super-p-brane theories [17, 18, 19, 20, 21, 22, 23, 24]. The nondegenerate supertranslation algebra is obtained by introducing a fermionic central extension, and the fermionic center together with a supercharge makes the nondegenerate group metric. This algebra may play a useful role in quantizing the theory as shown in the random lattice case [16]. In section 2 we construct an open superstring theory based on the nondegenerate supertranslation algebra, where fermionic and bosonic auxiliary fields carry boundary contributions.

For an open superstring there is an ambiguity in the GSO projection in the NSR formalism. In the GS formalism this ambiguity corresponds to an ambiguity in the boundary condition of fermionic coordinates [13]. A tachyonic state, which has the wrong sign for the GSO projection, is obtained by considering a superstring connecting a Dp-brane and an anti-Dp-brane $(\overline{\mathrm{Dp}})$. As an example if we take a superstring connecting a D2-brane and a $\overline{\mathrm{D} 2}$-brane, then its boundary condition is given by $\mathrm{U}$

$$
\begin{aligned}
& \Gamma_{012} \theta_{1}=\theta_{2}, \quad \text { at } \sigma=0 \\
& \Gamma_{012} \theta_{1}=-\theta_{2} \quad, \quad \text { at } \sigma=\pi .
\end{aligned}
$$

By defining $\theta(\sigma)$ on $-\pi \leq \sigma \leq \pi$ by $\theta(\sigma)=\Gamma_{012} \theta_{1}(-\sigma)$ on $-\pi \leq \sigma \leq 0$ and $\theta(\sigma)=\theta_{2}(\sigma)$ on $0 \leq \sigma \leq \pi, \theta$ is antiperiodic, $\theta(\pi)=-\theta(-\pi)$. There is no global SUSY parameter satisfying (1.3) since $\theta(\sigma)$ has no zero mode. This boundary condition still consistently cancels the surface term from the variation of the light-cone gauge fixed action

$$
\bar{\theta}_{1} \Gamma^{-} \delta \theta_{1}-\bar{\theta}_{2} \Gamma^{-} \delta \theta_{2}=\bar{\theta}_{1} \Gamma^{-} \delta \theta_{1}-\left(\mp \bar{\theta}_{1} \Gamma_{210}\right) \Gamma^{-}\left( \pm \Gamma_{012} \delta \theta_{1}\right)=0
$$

where $\Gamma^{ \pm}=\left(\Gamma^{0} \pm \Gamma^{1}\right) / \sqrt{2}$ and $\Gamma^{+} \theta_{1,2}=0$.

It turns out that the antiperiodic boundary condition on the fermionic coordinates (1.3) leads to a non-zero value of the fermionic central charge as we will see in section 3. We will also see that the mass must have an imaginary value. The non-BPS superalgebra, which has no zero eigenvalue of $\left\{Q_{\alpha}, Q_{\beta}\right\}$, is obtained by introducing an imaginary value for the tachyonic string energy realizing complete SUSY breaking. This choice corresponds to the wrong sign for the GSO projection.

In section 4, we demonstrate the D2- $\overline{\mathrm{D} 2}$ annihilation scenario from the superalgebra point of view. Depending on the configuration of the gauge fields the D2- and $\overline{\mathrm{D} 2}$-branes may annihilate into nothing [25] or into D0-branes [26, 12]. After passing through unstable non-BPS states the system settles down in the stable BPS state. The superalgebra gives a simple description of the brane charge cancellation and the cancellation of the brane tensions and the tachyon potential.

$$
{ }^{1} \Gamma^{11}=\Gamma^{\natural}, \Gamma^{\natural}\left(\begin{array}{c}
\epsilon_{1} \\
\epsilon_{2}
\end{array}\right)=\left(\begin{array}{c}
\epsilon_{1} \\
-\epsilon_{2}
\end{array}\right) \text { and } \Gamma_{012}=\Gamma_{[0} \Gamma_{1} \Gamma_{2]} / 3 ! \text {. }
$$




\section{Open superstring theory}

In this section we will obtain the gauge invariant and SUSY invariant Wess-Zumino action for an open superstring ([1.2). We first construct the nondegenerate supertranslation algebra for type IIA as an example and then calculate the SUSY invariant Maurer-Cartan (MC) one-forms $L$, and find a two-form $\mathcal{B}$ whose derivative gives the three-form $H=d \mathcal{B}$. Since the DBI gauge fields, with which the end points interact, live in the branes rather than in the string world-sheet, the interaction is not written in terms of the string worldsheet fields. Instead of using gauge fields in the Wess-Zumino action, we enlarge the superspace and introduce auxiliary coordinates which correspond to endpoint degrees of freedom.

The type IIA nondegenerate superalgebra [18] for an open superstring is

$$
\begin{aligned}
& \left\{Q_{\alpha}, Q_{\beta}\right\}=2\left(C \not P+C \Gamma^{\natural} \psi\right)_{\alpha \beta} \\
& {\left[P_{m}, Q_{\alpha}\right]=i\left(Z \Gamma_{m}\right)_{\alpha}} \\
& {\left[\Sigma_{m}, Q_{\alpha}\right]=i\left(Z \Gamma^{\natural} \Gamma_{m}\right)_{\alpha},}
\end{aligned}
$$

which is determined from the 10-dimensional IIA cyclic identity

$$
\left(\Gamma_{m}\right)_{(\beta}^{\alpha}\left(C \Gamma^{m}\right)_{\gamma \delta)}+\left(\Gamma^{\natural} \Gamma_{m}\right)_{(\beta}^{\alpha}\left(C \Gamma^{\natural} \Gamma^{m}\right)_{\gamma \delta)}=0 \quad, \quad \Gamma^{\natural}=\Gamma^{11} .
$$

The type IIB theory is obtained by replacing $\Gamma^{\natural}$ by $\tau_{3}$ acting on $\mathrm{N}=2$ fermion index. The enlarged superspace coordinates are introduced as parameters of the group element

$$
g=e^{i \xi^{\alpha} Z_{\alpha}} e^{i Y^{m} \Sigma_{m}} e^{i X^{m} P_{m}} e^{i \theta^{\alpha} Q_{\alpha}}
$$

and the MC one-forms are obtained as

$$
\begin{aligned}
& g^{-1} d g=i\left(\mathbf{L}^{m} P_{m}+L^{\alpha} Q_{\alpha}+\mathbf{L}_{\Sigma}^{m} \Sigma_{m}+L_{Z}^{\alpha} Z_{\alpha}\right) \\
& \left\{\begin{aligned}
\mathbf{L}^{m} & =d X^{m}-i \bar{\theta} \Gamma^{m} d \theta \\
L^{\alpha} & =d \theta^{\alpha} \\
\mathbf{L}_{\Sigma}^{m} & =d Y^{m}-i \bar{\theta} \Gamma^{\natural} \Gamma^{m} d \theta \\
L_{Z}^{\alpha} & =d \xi^{\alpha}+\left(\Gamma_{m} \theta\right)^{\alpha}\left(d X^{m}-i \frac{1}{3} \bar{\theta} \Gamma^{m} d \theta\right) \\
& +\left(\Gamma^{\natural} \Gamma_{m} \theta\right)^{\alpha}\left(d Y^{m}-i \frac{1}{3} \bar{\theta} \Gamma^{\natural} \Gamma^{m} d \theta\right)
\end{aligned}\right.
\end{aligned}
$$

which satisfies the MC equations

$$
\begin{aligned}
& d \mathbf{L}^{m}+i \bar{L} \Gamma^{m} L=0 \quad, \quad d L=0 \\
& d \mathbf{L}_{\Sigma}^{m}+i \bar{L} \Gamma^{\natural} \Gamma^{m} L=0 \quad, \quad d L_{Z}-\mathbf{Z} L-\Gamma^{\natural} \mathbf{Z}_{\Sigma} L=0 .
\end{aligned}
$$

The NS two-form $\mathcal{B}$ whose derivative becomes closed three-form $H$ is given by

$$
\begin{aligned}
d \mathcal{B} & =H=\bar{L} \Gamma^{\natural} \mathbf{\Psi} L \\
\mathcal{B} & =\frac{1}{2}\left(\bar{L} \Gamma^{\natural} L_{Z}-i \mathbf{L} \cdot \mathbf{L}_{\Sigma}\right),
\end{aligned}
$$


and this is the combination which enters into the SUSY invariant Wess-Zumino action.

An action for the open superstring theory of type IIA is

$$
\begin{aligned}
I_{\mathrm{F} 1^{\prime}} & =\int d^{2} \sigma\left(\mathcal{L}_{N G}+\mathcal{L}_{W Z}\right) \\
\mathcal{L}_{N G} & =-T \sqrt{-\operatorname{det} G_{\mu \nu}}, \quad G_{\mu \nu}=\mathbf{L}_{\mu} \cdot \mathbf{L}_{\nu} \\
\mathcal{L}_{W Z} & =-\frac{i}{2} T \epsilon^{\mu \nu} \mathcal{B}_{\mu \nu} \quad, \quad \mathcal{B}_{\mu \nu}=-\frac{1}{2}\left(\bar{L}_{\mu} \Gamma^{\natural} L_{Z \nu}+i \mathbf{L}_{\mu} \cdot \mathbf{L}_{\Sigma \nu}\right)-(\mu \leftrightarrow \nu)
\end{aligned}
$$

where $\sigma^{\mu}$ are worldsheet coordinates with $\mu=0,1$. The two form, $\mathcal{B}=\frac{1}{2} d \sigma^{\mu} d \sigma^{\nu} \mathcal{B}_{\mu \nu}$, is given by (2.8) and

$$
\begin{aligned}
& \mathcal{B}_{\mu \nu}=B_{\mu \nu}-\partial_{[\mu} A_{\nu]} \\
& \left\{\begin{array}{c}
B_{\mu \nu}=\partial_{\mu} \bar{\theta} \Gamma^{\natural} \Gamma^{m} \theta\left(\partial_{\nu} X_{m}-i \frac{1}{2} \partial_{\nu} \bar{\theta} \Gamma_{m} \theta\right)-(\mu \leftrightarrow \nu) \\
A_{\nu}=\frac{1}{2}\left(\bar{\theta} \Gamma^{\natural} \partial_{\nu} \xi+i X^{m} \partial_{\nu} Y_{m}\right)
\end{array}\right.
\end{aligned}
$$

The constraint set of the system is obtained as

$$
\begin{aligned}
H= & \tilde{p}^{2}+T^{2} G_{11}=0 \\
H_{\perp}= & \tilde{p} \cdot \mathbf{L}_{1}=0 \\
F= & \zeta-i \bar{\theta}\left(\tilde{p}-\frac{T}{2} \mathbf{L}_{\Sigma 1}+\frac{T}{2} \Gamma^{\natural} L_{1}\right)+i \frac{T}{2} \bar{L}_{Z 1} \Gamma^{\natural} \\
& +\frac{T}{6}\left(\bar{L}_{1} \Gamma^{\natural} \Gamma \theta \cdot \bar{\theta} \Gamma+\bar{L}_{1} \Gamma \theta \cdot \bar{\theta} \Gamma^{\natural} \Gamma\right)=0 \\
\phi_{Y}= & p_{Y}-\frac{T}{2}\left(\mathbf{L}_{1}+i \bar{L}_{1} \Gamma \theta\right)=p_{Y}-\frac{T}{2} X^{\prime}=0 \\
\phi_{\xi}= & \pi_{\xi}+i \frac{T}{2} \bar{L}_{1} \Gamma^{\natural}=\pi_{\xi}+i \frac{T}{2} \bar{\theta}^{\prime} \Gamma^{\natural}=0
\end{aligned}
$$

for canonical pairs $\left(X^{m}, \theta^{\alpha}, Y^{m}, \xi^{\alpha}\right)$ and $\left(p_{m}, \zeta_{\alpha}, p_{Y m}, \pi_{\xi \alpha}\right)$ and where $\tilde{p}$ is the SUSY invariant combination

$$
\tilde{p}_{m}=p_{m}+\frac{T}{2} \mathbf{L}_{\Sigma 1 m}-i \frac{T}{2} \bar{L}_{1} \Gamma^{\natural} \Gamma_{m} \theta=p_{m}+\frac{T}{2} Y_{m}^{\prime}+i T \bar{\theta} \Gamma^{\natural} \Gamma_{m} \theta^{\prime}
$$

The constraint set is invariant under the global SUSY transformation (2.21) up to constraints. As in 24] the fermionic constraints (2.14) satisfies the following algebra

$$
\left\{F_{\alpha}(\sigma), F_{\beta}\left(\sigma^{\prime}\right)\right\}=-2 i\left(C \Xi_{+}\right)_{\alpha \beta} \delta\left(\sigma-\sigma^{\prime}\right) \quad, \quad \Xi_{ \pm}=\Gamma^{m} \tilde{p}_{m} \pm T \Gamma^{\natural} \Gamma^{m} \mathbf{L}_{1 m}
$$

One half of $F_{\alpha}$ are first class constraints generating the kappa symmetry with generator given by

$$
\tilde{F}_{\alpha}=\left(F \Xi_{-}\right)_{\alpha}=0 \quad, \quad \Xi_{+} \Xi_{-} \approx 0
$$

It is important to examine behavior of the endpoints. For simplicity, let us consider the ground state in which fermionic variables can be neglected. For the Neumann boundary 
directions denoted by $\mu, \mathbf{L}_{1}^{\mu}=0$ is satisfied at boundaries. For the Dirichlet boundary directions denoted by $i, \mathbf{L}_{1}^{i} \neq 0$ at boundaries is obtained. From the constraint (2.13) it follows that $\tilde{p}^{i}=0$. The $\tau$ diffeomorphism constraint (2.12) at the boundaries is written as

$$
H=\left(\tilde{p}^{\mu}\right)^{2}+T^{2}\left(X^{\prime i}\right)^{2}=0 .
$$

The Dirichlet boundary gives rise to a mass $T$. As a result massive endpoints are moving on D2-branes.

The SUSY transformations are given by

$$
\left\{\begin{array}{l}
\delta \theta^{\alpha}=\epsilon^{\alpha} \\
\delta X^{m}=-i \bar{\epsilon} \Gamma^{m} \theta \\
\delta Y^{m}=-i \bar{\epsilon} \Gamma^{\natural} \Gamma^{m} \theta \\
\delta \xi^{\alpha}=X \epsilon^{\alpha}+\Gamma^{\natural} y / \epsilon^{\alpha}-\frac{i}{3}\left(\Gamma \theta^{\alpha} \cdot \bar{\epsilon} \Gamma \theta+\Gamma^{\natural} \Gamma \theta^{\alpha} \cdot \bar{\epsilon} \Gamma^{\natural} \Gamma \theta\right)
\end{array},\right.
$$

under which the MC one-forms (2.6) are invariant. The general form of the supercharges is

$$
Q \epsilon=\int d \sigma\left(p \cdot \delta X+\zeta \delta \theta+p_{Y} \cdot \delta Y+\pi_{\xi} \delta \xi\right)
$$

Using (2.21) the supercharges are obtained as

$$
Q_{\alpha}=\int d \sigma\left[\zeta+i \overline{\theta_{p}} \not+i \bar{\theta} \Gamma^{\natural} \not d_{Y}+\pi_{\xi}\left(X+\Gamma^{\natural} Y /\right)+i \frac{1}{3}\left(\pi_{\xi} \Gamma \theta \cdot \bar{\theta} \Gamma+\pi_{\xi} \Gamma^{\natural} \Gamma \theta \cdot \bar{\theta} \Gamma^{\natural} \Gamma\right)\right]_{\alpha}
$$

which satisfy the expected superalgebra (2.1)

$$
\left\{Q_{\alpha}, Q_{\beta}\right\}=2 i\left(C P P+C \Gamma^{h} \not\right)_{\alpha \beta} .
$$

The factor of $i$ on the right hand side arises by the usual replacement of the commutator, $\left[X^{m}(\sigma), P_{n}\left(\sigma^{\prime}\right)\right]=-i \delta_{n}^{m} \delta\left(\sigma-\sigma^{\prime}\right)$, by the Poisson bracket, $\left\{X^{m}(\sigma), P_{n}\left(\sigma^{\prime}\right)\right\}=\delta_{n}^{m} \delta\left(\sigma-\sigma^{\prime}\right)$.

Other charges are written in terms of canonical variables

$$
\begin{aligned}
P_{m} & =\int d \sigma p_{m} \\
\Sigma_{m} & =\int d \sigma p_{Y m} \approx \frac{T}{2} \int d \sigma X_{m}^{\prime}=-\frac{T}{2}\left(\left.X_{m}\right|_{\sigma=0}-\left.X_{m}\right|_{\sigma=\pi}\right) \\
Z_{\alpha} & =\int d \sigma \pi_{\xi \alpha} \approx-i \frac{T}{2} \int d \sigma \bar{\theta}^{\prime} \Gamma_{\alpha}^{\natural}=i \frac{T}{2}\left(\left.\bar{\theta} \Gamma_{\alpha}^{\natural}\right|_{\sigma=0}-\left.\bar{\theta} \Gamma_{\alpha}^{\natural}\right|_{\sigma=\pi}\right)
\end{aligned}
$$

where constraints (2.15) and (2.16) are used. The periodic boundary condition gives

$$
Z_{\alpha}=0
$$

This is consistent with the algebra (2.2) and (2.3) in the presence of D-branes where $P_{i}=0, \Sigma_{i}=0, i=4, \ldots, 9$ in the Dirichlet directions. 
If we use the constraint (2.15), the BPS condition obtained from the superalgebra (2.1) is different from the expected one; the BPS mass is half of the expected value, $P_{0}=T d / 2$. The origin of factor $1 / 2$ is the coefficient of the Wess-Zumino action which is determined by the kappa symmetry (2.18) and (2.19). To avoid confusion, note that this mass, $P_{0}=T d / 2$, is the mass of an open superstring attached to two branes including the effect of the auxiliary fields. If we impose $P_{0}=T d$ the coefficient of the Wess-Zumino action should be a factor of 2 larger. This breaks the kappa symmetry and there are twice as many fermionic variables. This phenomenon is a property of the world-volume action for non-BPS branes in [30]. In this paper we will consider a situation in which the BPS condition is modified in a manner that preserves the kappa symmetry. This will allow an efficient first-quantized description of tachyon condensation.

\section{The tachyonic superstring}

In this section we focus on an open superstring connecting D2- and $\overline{\mathrm{D} 2}$-branes with separation $d$. The fermionic coordinates $\theta$ satisfy (1.3) which is an antiperiodic boundary condition. It is interesting to consider this situation from the superalgebra point of view. We begin with the nondegenerate supertranslation algebra for an open superstring (2.1), (2.2) and (2.3).

When the antiperiodic boundary condition (1.3) is imposed, the fermionic central charges cannot be set to be zero;

$$
\begin{aligned}
Z_{A \alpha} & \equiv-i \frac{T}{2}\left(\left.\bar{\theta}_{A} \Gamma_{\alpha}^{\natural}\right|_{\sigma=0}-\left.\bar{\theta}_{A} \Gamma_{\alpha}^{\natural}\right|_{\sigma=\pi}\right) \\
Z_{1 \alpha} & =0, \rightarrow Z_{2 \alpha} \neq 0 .
\end{aligned}
$$

The nonvanishing fermionic center $Z_{\alpha}$ in (3.1) is crucial in this non-BPS system in contrast with (2.28) which describes a BPS system. This nonvanishing property leads to an inconsistency in the algebra (2.2) and (2.3) or equivalently

$$
\begin{aligned}
& {\left[P_{m}-\Sigma_{m}, Q_{1}\right]=2 i Z_{1} \Gamma_{m}=0} \\
& {\left[P_{m}+\Sigma_{m}, Q_{2}\right]=2 i Z_{2} \Gamma_{m} \neq 0 .}
\end{aligned}
$$

Although the right hand sides of (3.2) are non-zero, the Dirichlet components of the left hand side are zero for $d \rightarrow 0$ because $P_{i}=0$ and $\Sigma_{i}=0, i=3, \ldots, 9$. In other words, by taking the limit $d \rightarrow 0$, the antiperiodic boundary condition on $\theta$ 's, twisting fermions, causes a singularity in the superspace. One resolution of this inconsistency is to consider complex $P_{m}$, which allows for the presence of a tachyonic open string state. It is expected that this singularity is resolved by quantum excitation of such tachyonic modes. Collective tachyonic excitation makes the tachyon potential which contributes to the energy, $P_{0}$. This will be discussed in the next section. In this section we concentrate on the algebraic consistency, where we introduce "tachyonic modes" in the algebra as "imaginary" mass, $P=\Re P+i \Im P \neq 0$. So the left hand side of (3.2) becomes non-zero, 
$(P+\Sigma)=\Re(P+\Sigma)+i \Im P \neq 0$. The BPS condition should now be examined carefully, by considering the superalgebra (2.1) for a string lying along $X^{3}$ direction at rest

$$
\{Q, Q\} \epsilon=2\left(P_{0}-\frac{T d}{2} \Gamma^{\natural 03}\right) \epsilon=0 .
$$

With imaginary $P_{m}$, say $P_{0}=i m_{T}$ with real $m_{T}$,

$$
\operatorname{det}\left(i m_{T}-\frac{T d}{2} \Gamma^{\natural 03}\right) \neq 0
$$

so the only solution of (3.3) is $\epsilon=0$ and SUSY is completely broken. In this way the antiperiodic boundary condition leads to a non-BPS algebra. Note that in this classical approach, the value of the first quantized tachyonic mass $m_{\text {Tachyon }}^{2}=-\frac{1}{2 \alpha^{\prime}}$ cannot be determined.

It is important that there is a supercharge (2.23) under which the action is invariant not only for the usual fundamental open superstring but also a tachyonic superstring. The use of auxiliary fields is essential here to make a self-consistent open string theory. Without introducing local auxiliary fields, the global symmetries of the open string action depend on the boundary interactions. By using auxiliary fields the SUSY invariance can be guaranteed at least at the level of the action, independent of the boundaries. The difference between the usual superstring and the tachyonic superstring is "the expectation value" of the fermionic central charge $Z_{\alpha}$ and momentum $P_{m}$. Once the vacuum is chosen, "the expectation value" is fixed and the extended superalgebra (2.1), (2.2) and (2.3) leads to BPS states or non-BPS states as shown here.

\section{The $\mathrm{Dp}-\overline{\mathrm{Dp}}$ system}

Now let us consider the Dp-F1- $\overline{\mathrm{Dp}}$ system. As an example we consider the following configuration of type IIA theory: A D2-brane and a $\overline{\mathrm{D} 2}$-brane lying in the $X^{1}-X^{2}$ directions, and an open superstring with Dirichlet boundaries $X^{i}=0$ with $i=3, \ldots, 9$ at $\sigma=0$ and $X^{3}=d$ and $X^{i}=0$ with $i=4, \ldots, 9$ at $\sigma=\pi$. The total action of this system is given by the sum of actions for a D2-brane, a $\overline{\mathrm{D} 2}$-brane and an open superstring (F1) and their interactions:

$$
\begin{aligned}
I_{\text {total }} & =I_{\mathrm{D} 2}\left[A_{\mu}\right]+I_{\overline{\mathrm{D}} 2}\left[\bar{A}_{\mu}\right]+I_{\mathrm{F} 1}+I_{\text {int }}\left[A_{\mu}, \bar{A}_{\mu}\right] \\
I_{\text {int }} & =\left.\int_{\mathrm{D} 2} d \tau A_{\mu} j^{\mu}\right|_{\sigma=0}-\left.\int_{\overline{\mathrm{D} 2}} d \tau \bar{A}_{\mu} j^{\mu}\right|_{\sigma=\pi} .
\end{aligned}
$$

The end points of an open superstring are sources of DBI gauge fields, so the Gauss law constraints become

$$
\partial_{a} E^{a}=\left.j^{0}\right|_{\sigma=0}=T \delta^{(3)}(x-X(\tau)), \partial_{a} \bar{E}^{a}=-\left.j^{0}\right|_{\sigma=\pi}=-T \delta^{(3)}(x-X(\tau)), a=1,2(4.2)
$$

where the canonical conjugates of $A_{\mu}, \bar{A}_{\mu}$ are $E^{\mu}, \bar{E}^{\mu}, \mu=0,1,2$. Under a global SUSY transformation, $I_{\mathrm{D} 2}, I_{\overline{\mathrm{D}} 2}$ and $I_{\mathrm{F} 1}+I_{\text {int }}$ are invariant. Although the Wess-Zumino action of 
an open superstring $I_{\mathrm{F} 1}$ gives rise to a nonvanishing surface term, the variation of the DBI gauge fields in the interaction $I_{\text {int }}$ cancels it. We identify this SUSY invariant combination $I_{\mathrm{F} 1}+I_{\text {int }}$ with the self-contained open superstring action $I_{\mathrm{F} 1^{\prime}}(2.10)$. Auxiliary fields in $I_{\mathrm{F} 1^{\prime}}$ are related to the DBI gauge fields by the relation (2.11). It may be confusing that there exist Wess-Zumino actions not only for an open superstring but also Dp, $\overline{\mathrm{Dp}}$ branes, but in this paper we modify only a Wess-Zumino action for an open superstring and keep usual forms of Wess-Zumino actions for Dp, $\overline{\mathrm{Dp}}$ branes which have usual boundary conditions.

Each Noether charge on world-volume is written in terms of world-volume variables, so different world-volume charges commute/anticommute as long as the world-volumes are separated. The superalgebra of a D2-brane is given by [29],

$$
\begin{aligned}
\left\{Q_{\mathrm{D} 2 \alpha}, Q_{\mathrm{D} 2 \beta}\right\}= & 2 i\left(C \not P-T_{D 2} C \int \partial_{1} X \partial_{2} X\right)_{\alpha \beta}+2 i\left(C \Gamma^{\natural} \int \partial_{\alpha} X E^{a}-C \Gamma^{\natural} T_{D 2} \int F_{12}\right)_{\alpha \beta} \\
& +\frac{i}{2} \int \partial_{a} E^{a}\left(\bar{\theta} \Gamma^{\natural} \Gamma_{\alpha} \cdot \bar{\theta} \Gamma_{\beta}+\bar{\theta} \Gamma^{\natural} \Gamma_{\beta} \cdot \bar{\theta} \Gamma_{\alpha}\right)
\end{aligned}
$$

An anti-BPS state is obtained by reversing the brane charge, or equivalently by taking opposite sign for the world-volume coordinate, for example

$$
\begin{aligned}
& \int_{\overline{\mathrm{D} 2}} d^{2} \sigma \epsilon^{a b} T_{\mathrm{D} 2} \partial_{a} X \partial_{b} X=-\int d^{2} \sigma \epsilon^{a b} T_{\mathrm{D} 2} \partial_{a} X \partial_{b} X=-T_{\mathrm{D} 2} V \\
& \int_{\overline{\mathrm{D} 2}} d^{2} \sigma \epsilon^{a b} F_{a b}=-\int d^{2} \sigma \epsilon^{a b} F_{a b} .
\end{aligned}
$$

The superalgebra for an anti-D2-brane is given by

$$
\begin{aligned}
\left\{Q_{\overline{\mathrm{D} 2} \alpha}, Q_{\overline{\mathrm{D} 2} \beta}\right\}= & 2 i\left(C \not P-T_{D 2} C \int_{\overline{\mathrm{D} 2}} \partial_{1} X \partial_{2} X\right)_{\alpha \beta}+2 i\left(C \Gamma^{\natural} \int_{\overline{\mathrm{D} 2}} \partial_{a} X \bar{E}^{a}-C \Gamma^{\natural} T_{D 2} \int_{\overline{\mathrm{D} 2}} \bar{F}_{12}\right)_{\alpha \beta} \\
& +\frac{i}{2} \int_{\overline{\mathrm{D} 2}} \partial_{a} \bar{E}^{a}\left(\bar{\theta} \Gamma^{\natural} \Gamma_{\alpha} \cdot \bar{\theta} \Gamma_{\beta}+\bar{\theta} \Gamma^{\natural} \Gamma_{\beta} \cdot \bar{\theta} \Gamma_{\alpha}\right) \\
= & 2 i\left(C \not P+T_{D 2} C \int \partial_{1} X \partial_{2} X\right)_{\alpha \beta}+2 i\left(C \Gamma^{\natural} \int \partial_{a} X \bar{E}^{a}+C \Gamma^{\natural} T_{D 2} \int \bar{F}_{12}\right)_{\alpha \beta} \\
& +\frac{i}{2} \int \partial_{a} \bar{E}^{a}\left(\bar{\theta} \Gamma^{\natural} \Gamma_{\alpha} \cdot \bar{\theta} \Gamma_{\beta}+\bar{\theta} \Gamma^{\natural} \Gamma_{\beta} \cdot \bar{\theta} \Gamma_{\alpha}\right) .
\end{aligned}
$$

The expression for the superalgebras for other Dp-brane systems are given in [27, 28, 29].

We focus on one superstring connecting unit volumes of the D2-brane and $\overline{\mathrm{D} 2}$-brane. The total supercharge is

$$
Q=Q_{\mathrm{D} 2}+Q_{\overline{\mathrm{D} 2}}+Q_{\mathrm{F} 1^{\prime}}
$$

First we look for a case where the DBI gauge fields do not have any topological excitation. The anticommutator of the total supercharges (4.6) is

$$
\begin{aligned}
\{Q, Q\} & =\left\{Q_{\mathrm{D} 2}, Q_{\mathrm{D} 2}\right\}+\left\{Q_{\overline{\mathrm{D} 2}}, Q_{\overline{\mathrm{D} 2}}\right\}+\left\{Q_{\mathrm{F} 1^{\prime}}, Q_{\mathrm{F} 1^{\prime}}\right\} \\
& =2\left(\left(P_{\mathrm{D} 2,0}+P_{\overline{\mathrm{D} 2}, 0}+P_{F 1^{\prime}, 0}\right)-\left(T_{\mathrm{D} 2}-T_{\mathrm{D} 2}\right) \Gamma^{012}+\frac{T d}{2} \Gamma^{\natural 03}\right)
\end{aligned}
$$


The D2- and $\overline{\mathrm{D} 2}$-brane charges in the second term cancel. The $F 1^{\prime}$ charge in the third term vanishes in the limit $d \rightarrow 0$. When we bring the $\overline{\mathrm{D} 2}$-brane close to the D2-brane $(d \rightarrow 0)$, many tachyonic modes are excited and form the tachyon potential. We treat one tachyonic superstring in the background of many other tachyonic superstrings as a string in a tachyon potential, using the mean field approximation. Whereas in section 3 we considered a single tachyonic superstring we will now discuss a tachyonic string in the presence of a tachyonic potential in order to describe tachyon condensation. The energy for an isolated tachyonic string, $P_{\mathrm{F} 1^{\prime}, 0}=i m_{T}$, corresponds to the top of the local maximum of the tachyon potential, while the energy in the presence of $N$ tachyonic strings is $\sum^{N} P_{\mathrm{F}^{\prime}, 0}=N \mathcal{V}_{\min }(\mathcal{T})$ which corresponds to the local minimum of the tachyon potential. The average energy of such a tachyonic superstring is

$$
P_{\mathrm{F} 1^{\prime}, 0} \longrightarrow \mathcal{V}_{\min }(\mathcal{T})
$$

The energy per unit volume of the D2-( $\overline{\mathrm{D} 2}-)$ brane is $P_{D 2,0}=T_{D 2}\left(P_{\overline{\mathrm{D} 2}, 0}=T_{D 2}\right)$. The condition of the first term in (4.7)

$$
P_{\mathrm{D} 2,0}+P_{\overline{\mathrm{D} 2}, 0}+P_{\mathrm{F} 1^{\prime}, 0}=2 T_{\mathrm{D} 2}+\mathcal{V}_{\min }(\mathcal{T})=0
$$

describes the situation in which a D2- $\overline{\mathrm{D} 2}$ pair annihilates.

Next the gauge field contribution to the superalgebra (4.7) is taken into account;

$$
\begin{aligned}
\left\{Q_{\alpha}, Q_{\beta}\right\}= & 2\left(\left(P_{\mathrm{D} 2,0}+P_{\overline{\mathrm{D} 2}, 0}+P_{F 1^{\prime}, 0}\right)-\left(T_{\mathrm{D} 2}-T_{\mathrm{D} 2}\right) \Gamma^{012}+\frac{T d}{2} \Gamma^{\natural 03}\right) \\
& +2\left(\Gamma^{0 \natural a} \int\left(E^{a}+\bar{E}^{a}\right)-\Gamma^{0 \natural} T_{\mathrm{D} 2} \int\left(F_{12}-\bar{F}_{12}\right)\right) \\
& +\int \partial_{a}\left(E^{a}+\bar{E}^{a}\right) \frac{i}{2}\left(\bar{\theta} \Gamma^{\natural} \Gamma_{\alpha} \cdot \bar{\theta} \Gamma_{\beta}+\bar{\theta} \Gamma^{\natural} \Gamma_{\beta} \cdot \bar{\theta} \Gamma_{\alpha}\right) .
\end{aligned}
$$

After computing the algebra, the integral on the right hand side of (4.10) can be written as a 2-dimensional world-volume integral. When the vortex configuration occurs in the tachyon fields, $\mathcal{T}=|\mathcal{T}| e^{i n \chi}$, the magnetic fields on the D2-branes take the following configurations

$$
F_{12}-\bar{F}_{12}=\frac{1}{2 \pi}\left[\partial_{1}, \partial_{2}\right] \ln \mathcal{T}
$$

This tachyon vortex configuration does not give an electric configuration, so we may set $E^{a}+\bar{E}^{a}=0$. However, it is also interesting to consider nontrivial configurations of $E+\bar{E}$ [31]. By taking the $2+1$ space-time dual, $\epsilon_{a b}\left(E^{a}+\bar{E}^{a}\right)=\partial_{b}(\varphi-\bar{\varphi})$, scalar fields $\varphi$ and $\bar{\varphi}$ appear which correspond to 11-th dimensional coordinates. So nonzero $\int\left(E^{a}+\bar{E}^{a}\right)$ means nontrivial winding of the relative D2- $\overline{\mathrm{D} 2}(\mathrm{M} 2-\overline{\mathrm{M}} 2)$ on the compact 11-th dimensional direction. Since the term $\Gamma^{0 \natural a} \int\left(E^{a}+\bar{E}^{a}\right)$ in (4.10) is universal for arbitrary Dp-branes [27, 28, 29], the superalgebras for an arbitrary Dp- $\overline{\mathrm{Dp}}$ system are sensitive to this type of winding effect. 
As a result of using (4.11) the superalgebra is

$$
\{Q, Q\}=2\left(P_{0}-n T_{\mathrm{D} 2} \Gamma^{0 \natural}\right),
$$

which is nothing but the superalgebra for $n$ D0-branes. Half the SUSY is recovered, and the final state is a BPS state.

\section{Discussion}

We have shown that the nondegenerate supertranslation algebra is suitable for representing an open superstring including the tachyonic superstring. The supercharge can be defined, taking into account the leakage of the supercurrent which is compensated by fermionic and bosonic auxiliary fields. For a tachyonic superstring the antiperiodic boundary condition on fermionic coordinates leads to a nonzero fermionic central charge and it turns out that imaginary valued momentum is required. In other words, both the fundamental superstring and the tachyonic superstring have the same symmetry structure and the difference is in the expectation value of the global charges. The GSO ambiguity in the NSR formalism corresponds to the ambiguity of the expectation values of momentum $P$ in the GS formalism as is seen for the BPS condition (3.3),

$$
\{Q, Q\} \epsilon=2\left(P_{0}-\frac{T d}{2} \Gamma^{\natural 03}\right) \epsilon=0
$$

This allows the following possibilities.

\begin{tabular}{c|c|c|c|c|c} 
& Mass & SUSY & $\left\{Q_{\alpha}, Q_{\beta}\right\}$ & $\theta$ boundary & GSO \\
\hline BPS & $P_{0}=\frac{T d}{2}$ & $\begin{array}{c}\epsilon=\Gamma^{\natural 03} \epsilon \\
\rightarrow^{\exists} \epsilon\end{array}$ & $\left(\begin{array}{cc}\mathbf{0} & \mathbf{0} \\
\mathbf{0} & \mathbf{2}\end{array}\right)$ & periodic & correct \\
\hline non-BPS & $P_{0}=i m_{T}$ & $\epsilon=0$ & $\operatorname{det}\left\{Q_{\alpha}, Q_{\beta}\right\} \neq 0$ & antiperiodic & wrong
\end{tabular}

We also showed that the superalgebra, defined by the sum of world-volumes of D2-F1'$\overline{\mathrm{D} 2}$, represents brane-antibrane annihilation. Without a topologically nontrivial configuration of tachyon fields, the brane-antibrane system which originally contains the non-BPS tachyonic superstring, reduces to nothing, which is the supersymmetric vacuum. If the tachyonic fields are in a vortex configuration, the brane-antibrane system reduces to the D0-brane system which is a BPS state. The merit of the superalgebra is that important combinations of charges of the system are automatically derived; such as the cancellation of the D-brane tensions, electromagnetic quantum numbers, $\int\left(F_{a b}-\bar{F}_{a b}\right)$ and $\int\left(E^{a}+\bar{E}^{a}\right)$. A problem of this approach is that the quantum tachyon mass or the field theoretical tachyon potential cannot be deteremined. This approach to the brane-antibrane system from the point of view of space-time SUSY is purely classical.

Applications of the non-degenerate superalgebra to manifestly supersymmetric open membrane theory and other p-brane theories are also possible [17]. The extension to nonabelian gauge interactions is an important issue. In this formulation the vector gauge 
interaction is represented by using auxiliary fields, so it is expected that the Chan-Paton factor is simply imposed on the auxiliary fields. This approach, where the space-time symmetry is manifest, will be useful for examining the conjecture that all branes can be obtained from the D9- and $\overline{\mathrm{D} 9}$-brane [12]. We leave these problems for a further publication.

\section{Acknowledgments}

We would like to express our gratitude to Michael B. Green for careful reading the manuscript and useful discussions. M.H. would like to thank Tamiaki Yoneya for a seminar given at KEK and for fruitful discussion, and she wishes to thank Ken-ji Hamada, Masashi Hayakawa and Nobuyuki Ishibashi for useful discussions. M.S. wishes to thank YITP and the theory group of KEK for the kind hospitality and gratefully acknowledges support by Nishina Memorial Foundation.

\section{References}

[1] E.Witten and D.Olive, Phys. Lett. B78 (1978) 97.

[2] E.Witten, Nucl. Phys. B460 (1996) 335, hep-th/9510135.

[3] J.Polchinski, TASI Lectures on D-branes, p.293 in Fields, Strings and Duality (TASI 96) eds. C.Efthimiou and B.Greene, World Scientific 1997, hep-th/9611050; J.Polchinski, S.Chaudhuri and C.Johnson, Notes on D-branes, hep-th/9602052.

[4] T.Banks and L.Susskind, Brane- anti-brane forces, hep-th/9511194.

[5] M.B.Green and M.Gutperle, Nucl. Phys. B476 (1996) 484, hep-th/9604091.

[6] A.Sen, An Introduction to Nonperturbative String Theory in Duality and supersymmetric theories p.297-413, Cambridge 1997, hep-th/9802051.

[7] O.Bergman and M.R.Gaberdiel, Phys. Lett. 441 (1998) 133,hep-th/9806155.

[8] A.Sen, J. High Energy Phys. 09 (1998) 023, hep-th/9808141.

[9] A.Sen, J. High Energy Phys. 10 (1999) 021, hep-th/9809111.

[10] A.Sen, J. High Energy Phys. 12 (1999) 021, hep-th/9812031.

[11] P.Horava, Adv. Theor. Math. Phys. 2 (1999) 1373, hep-th/9812135.

[12] E.Witten, J. High Energy Phys. 12 (1998) 019, hep-th/9810188.

[13] T.Yoneya, Nucl. Phys. B576 (2000) 219, hep-th/9912255.

[14] M.Kalb and P.Ramond, Phys. Rev. D9 (1974) 2273.

[15] M.B.Green, Phys. Lett. B223 (1989) 157.

[16] W.Siegel, Phys. Rev. D50 (1994) 2799, hep-th/9403144. 
[17] E.Bergshoeff and E.Sezgin, Phys. Lett. B354 (1995) 256, hep-th/9504140;

E.Sezgin, Phys. Lett. B392 (1997) 323, hep-th/9609086.

[18] M.Sakaguchi, Phys. Rev. D59 (1999) 046007, hep-th/9809113.

[19] M.Sakaguchi, J. High Energy Phys. 02 (1999) 017, hep-th/9811143.

[20] M.Abe, M.Hatsuda, K.Kamimura and T.Tokunaga, Nucl. Phys. B553 (1999) 305, hep-th/9903234.

[21] C.Chryssomalakos, J.A.de Azcarraga, J.M.Izquierdo, J.C.Perez Bueno, Nucl. Phys. B567 (2000) 293, hep-th/9904137.

[22] M.Sakaguchi, J. High Energy Phys. 04 (2000) 019, hep-th/9909143.

[23] M.Hatsuda and M.Sakaguchi, Nucl. Phys. B577 (2000) 183, hep-th/0001214.

[24] M.Hatsuda, K.Kamimura and M.Sakaguchi, Phys. Rev. D62 (2000) 105024, hepth/0007009.

[25] A.Sen, J. High Energy Phys. 08 (1998) 012, hep-th/9805170.

[26] A.Sen, J. High Energy Phys. 09 (1998) 023, hep-th/9808141.

[27] M.Hatsuda and K.Kamimura, Nucl. Phys. B520 (1998) 493, hep-th/9708001.

[28] K.Kamimura and M.Hatsuda, Nucl. Phys. B527 (1998) 381, hep-th/9712068.

[29] M.Hatsuda and K.Kamimura, Nucl. Phys. B535 (1998) 499, hep-th/9804087.

[30] A.Sen, J. High Energy Phys. 10 (1999) 008, hep-th/9909062.

[31] P.Yi, Nucl. Phys. B550 (1999) 214, hep-th/9901159;

O.Bergman, K.Hori and P.Yi, Nucl. Phys. B580 (2000) 289, hep-th/0002223. 